\title{
Les fidélités républicaines sous le Consulat et l'Empire
}

\section{Natalie Petiteau}

\section{(2) OpenEdition \\ 1 Journals}

Édition électronique

URL : https://journals.openedition.org/ahrf/7633

DOI : 10.4000/ahrf.7633

ISSN : 1952-403X

Éditeur :

Armand Colin, Société des études robespierristes

Édition imprimée

Date de publication : 1 décembre 2006

Pagination : 59-74

ISSN : 0003-4436

Référence électronique

Natalie Petiteau, «Les fidélités républicaines sous le Consulat et l'Empire », Annales historiques de la

Révolution française [En ligne], 346 | Octobre/Décembre 2006, mis en ligne le 01 décembre 2009, consulté le 23 avril 2022. URL : http://journals.openedition.org/ahrf/7633 ; DOI : https://doi.org/ 10.4000/ahrf.7633

Ce document a été généré automatiquement le 23 avril 2022.

Tous droits réservés 


\title{
Les fidélités républicaines sous le Consulat et l'Empire
}

\author{
Natalie Petiteau
}

1 S'interroger sur les fidélités républicaines de 1800 à 1815, c'est inviter à envisager l'histoire du Consulat et de l'Empire sous un angle peu habituel ${ }^{1}$. Car à suivre l'historiographie classique, tout se passerait comme si, dès les lendemains de Brumaire puis de l'attentat de la rue Saint-Nicaise, tous les républicains étaient soit ralliés, soit emprisonnés ou exilés. De plus, au premier rang des fidélités reniées, n'y a-t-il pas celle de Bonaparte lui-même? Républicain, il l'est lorsqu'il quitte la Corse en 1793, lorsqu'il porte secours au régime en vendémiaire ou lorsqu'il fonde des républiques sœurs en 1796. Républicain, il semble vouloir le demeurer même lorsque la Constitution de l'an XII proclame que le gouvernement de la République est confié à un empereur. Mais républicain, l'est-il encore au soir du coup d'État? Pourtant, et en dépit de cet illustre exemple de reniement, pourquoi l'idée républicaine disparaîtrait-elle sitôt proclamé le régime consulaire ? Comment se contenter de croire qu'elle ne survivrait qu'au travers de quelques meneurs finalement arrêtés, alors même que durant le premier XIX ${ }^{\mathrm{e}}$ siècle, la figure du vieux républicain conservant ses espoirs demeure une référence ${ }^{2}$. Certes, la teneur des archives de police n'est pas sans accréditer une telle interprétation : cellesci, après 1802, contiennent bien davantage de mentions explicites concernant les exchouans et parlent moins souvent d'anciens jacobins. Le régime demeurerait-il plus inquiet des agissements des royalistes que des menées des républicains ? En tout cas, on surveille activement les premiers, on mentionne de moins en moins les seconds. Preuve que ceux-ci ont été mis hors jeu quand ceux-là demeurent en mesure de s'opposer encore en raison des complaisances du régime à leur égard ${ }^{3}$ ? Il est vrai que dans les débuts du Consulat, la méfiance du régime s'exerce davantage, semble-t-il, à l'encontre des républicains que des royalistes.

2 Il serait dommageable d'envisager l'histoire du régime napoléonien sans poser la question du devenir des hommes et des femmes séduits par l'idée républicaine. Fort heureusement, les travaux menés récemment par les historiens de la Révolution portant désormais leur attention sur l'après $1799^{4}$ permettent aujourd'hui de ne plus 
oublier que les républicains ont encore une histoire après la Révolution. S'ils soulignent pour la plupart que les Barère, Antonelle, Félix Lepeletier ou Agricol Moureau font souvent figure de héros fatigués, ils mettent aussi en évidence le dynamisme d'un Rigomer Bazin ${ }^{5}$. Si bien qu'il est même présomptueux, aujourd'hui, de tenter d'y revenir, tant cette histoire, grâce à eux, est désormais bien écrite. Il demeure tout de même possible d'apporter de rares éléments nouveaux au dossier, en tentant de saisir notamment activités et devenir des républicains anonymes. Les rapports de police, les feuilles de travail par arrondissement, comme les bulletins quotidiens, qui ont fait l'objet des publications d'Alphonse Aulard, puis d'Ernest d'Hauterive et enfin de Nicole Gotteri, méritent en effet relecture en ce sens. On peut objecter que ces sources ne rendent compte que de ce que le régime veut bien surveiller, qu'elles laissent dans l'ombre les problèmes intérieurs quand les dangers extérieurs se font plus menaçants, qu'elles en disent peut-être plus sur la police que sur ceux que celle-ci surveille. Reste que, en dépit de leurs limites et par leurs défauts mêmes, ces sources ne doivent pas être négligées : elles conduisent, ne serait-ce qu'en étant attentif à la façon dont les policiers parlent de ce qu'ils surveillent, à saisir des traits essentiels de l'univers politique dans lequel évoluent les Français au quotidien. Elles permettent de se mettre parfois à l'écoute des paroles des humbles : même si celles-ci ne sont pas rapportées avec exactitude ou avec précision, l'historien trouve dans les rapports nombre d'indices précieux s'il fait l'effort d'une distance critique.

Des hommes sous surveillance

3 Tout au long des rapports de police, les opposants apparaissent souvent stigmatisés, rappelons-le, par le vocable d'« exclusifs ", à moins que ce ne soit celui d'« exagérés ", ou même encore d' " enragés ", puis d' " anarchistes " ${ }^{6}$ : le vocabulaire de la Révolution est ainsi conservé, il enferme les républicains dans la catégorie de l'opposition radicale $^{7}$, il les exclut d'emblée, par le sens même de ces mots, de cette République de l'extrême centre que Bonaparte semble incarner ${ }^{8}$. Or, la lecture de ces rapports laisse le sentiment que presque tous les faits et gestes de ces opposants républicains sont connus par infiltration de leurs réseaux. Ainsi, en septembre 1800, un rapport de police annonce qu'une grande réunion «d'exclusifs» doit avoir lieu "sous le prétexte de la franc-maçonnerie », mais il est prévu d'y introduire un agent qui connaît particulièrement bien ces hommes et qui les fréquente habituellement ${ }^{9}$. Le 4 novembre suivant, un nouveau rapport annonce l'arrestation de deux meneurs, Jumillard et Brissevin - plus tard déportés ${ }^{10}$-, mais la police continue d'en rechercher d'autres, butant toutefois sur le fait que "les hommes ne sortent presque plus; ce sont les femmes qui colportent les nouvelles et vont chercher de l'argent $»^{11}$. Aux mouchards de la police pénétrant dans l'espace du secret, les républicains répondent donc par l'organisation d'une sphère féminine encore anonyme aux yeux des policiers. L'existence même d'un espace politique clandestin favorise l'entrée des femmes en politique. Certes, leurs actions ne relèvent sans doute, pour partie d'entre elles, que de l'habituelle soumission à l'autorité masculine. Il n'en reste pas moins qu'elles apparaissent ainsi dans les rapports de police, et qu'elles se trouvent de ce fait à leur tour stigmatisées, jouissant donc d'une existence politique reconnue.

4 À la suite de l'attentat de la rue Saint-Nicaise, les arrestations se sont multipliées : 130 personnes sont inscrites sur une première liste de proscription ${ }^{12}, 98$ suspects sont définitivement déportés. Cette vague d'arrestations a permis de faire taire nombre d'opposants de faible envergure : elles concernent, note-t-on, "plusieurs exagérés » mais aussi des auteurs de simples «injures et menaces contre le gouvernement $»^{13}$. Ce 
qui n'empêche pas de repérer encore d'importantes réunions secrètes d'« enragés » au faubourg Saint-Antoine. L'attention de la police demeure soutenue afin de continuer les arrestations au moindre propos d'hostilité : or, de tels mots, qu'ils soient prononcés sur la place publique ou parfois même en cercle privé suffisent déjà à maintenir leur auteur en détention arbitraire. L'élimination progressive des républicains se fait dans l'absence totale de respect des libertés individuelles. Si fidélités républicaines il y a eu sous l'Empire, il ne faut pas oublier que cela s'est fait dans le cadre d'un régime policier recourant aux "mesures de haute police» pour enfermer des opposants sans jugement ${ }^{14}$. Si bien que l'on peut supposer que bien des républicains anonymes ont fini par taire leur fidélité : ainsi, ce champ politique peut être vu comme structuré en cercles successifs, des anonymes silencieux aux activistes déterminés en passant par ceux qui n'ont pas toujours su taire leurs convictions.

Mais, au début de 1802, les républicains sont moins souvent identifiés comme des « exagérés » ou des "exclusifs »: ils sont désignés de façon plus neutre du terme de «mécontents $»^{15}$. La police met désormais l'accent sur des oppositions apparemment individuelles, notant scrupuleusement les bruits colportés, mais sans se risquer à les attribuer à tel ou tel parti. Il faut également s'interroger sur le sens à donner au terme «malveillants » qui désormais apparaît à plusieurs reprises et semble bien désigner à son tour cette opposition républicaine ${ }^{16}$. Ainsi, en septembre 1802 , on note que «les malveillants répandent sourdement que l'on arrête des citoyens par ordres particuliers et immédiats du gouvernement ». Quelques jours plus tard, ces mêmes « malveillants » sont accusés de dire «que la guerre doit recommencer incessamment ${ }^{17}$. Et tandis que les royalistes continuent à exister comme tels dans les sources, les républicains se trouvent dilués dans cette opposition qui n'est même plus évaluée à l'aune des références révolutionnaires, ce qui est en fait un moyen de l'avilir.

6 Après quoi, à partir de l'automne de 1802, les rapports de police contiennent de nouveau de fréquentes rubriques consacrées aux « exclusifs » qui font toujours l'objet d'une très vigilante surveillance et dont l'opposition semble de nouveau fort inquiétante. En février 1803, depuis que le Corps législatif est de nouveau réuni, ils tiennent même de fréquentes réunions ${ }^{18}$; en juin, la police s'inquiète de les voir présents dans presque tous les cafés ${ }^{19}$. En septembre, ils apparaissent plus précisément cantonnés en quatre lieux, dont le Procope et une tabagie de la rue Saint-Germain l'Auxerrois, où ils disposent d'arrières-salles qui ne sont accessibles qu'aux personnes connues et reconnues par les membres du parti ${ }^{20}$. Il semble finalement qu'ils aient tenté un vaste mouvement lors de l'anniversaire de la proclamation de la République, en septembre $1803^{21}$, si bien que de nombreuses arrestations sont de nouveau opérées, preuve que l'épuration des lendemains de l'attentat de la rue Saint-Nicaise n'a pas éliminé tous les républicains. Du reste, cette nouvelle vague d'arrestations ne met pas non plus fin au mouvement, puisque des réunions se tiennent encore ${ }^{22}$. Reste que ces militants se sentent cernés de toutes parts à la fin d'octobre 1803 : ils disent alors

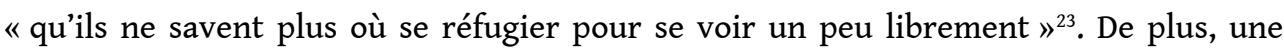
nouvelle vague d'arrestations est bientôt lancée, permettant de mettre la main sur des papiers qui éclairent la constitution des réseaux ${ }^{24}$. Si bien que lorsque le régime devient impérial, ces oppositions semblent à peu près étouffées.

7 D'ailleurs, à partir de 1804 , le vocabulaire évolue encore, et c'est sous le nom d'« anarchistes » qu'ils figurent désormais de plus en plus souvent dans les sources ${ }^{25}$, pour en disparaître à peu près en 1808. Dès lors, le régime est bien davantage 
préoccupé par les résistances à la conscription et la situation extérieure puis, à partir de 1810, par les mauvaises récoltes et la crise économique: la police semble moins attentive à livrer des rapports sur tous les signes d'oppositions intérieures. Cela est-il l'indice d'un réel recul des oppositions ou d'un véritable relâchement policier qui expliquerait l'affaire Malet? Quoi qu'il en soit, si Fouché a pu être soupçonné de protéger les jacobins ${ }^{26}$, ceux-ci ne sont pourtant pas davantage présents dans les bulletins de police avec sa disgrâce, comme si leur vivier s'était réellement tari ${ }^{27}$.

Des hommes au passé significatif

Les acteurs de la vie politique révolutionnaire figurent bien sûr aux premiers rangs des oppositions républicaines à l'Empire. Au début du Consulat, le mécontentement s'exprime, on le sait, autour des membres du Tribunat ou même du Corps législatif qui " commencent à s'ennuyer du silence qu'ils sont obligés de garder ». Il ne s'agit pas ici de revenir sur les oppositions malgré tout exprimées au sein des Assemblées: on sait notamment le courage de Carnot face à la proclamation de l'Empire, mais on sait aussi son isolement. Pourtant, la police repère nombre de "réunions secrètes " visant à organiser des protestations contre les décisions du gouvernement et à «s'assembler toutes les fois que les circonstances l'exigeront ", cela dans le but d'obtenir, espère-ton, la tenue de séances extraordinaires dans les Assemblées. On demeure donc bien là dans une logique républicaine, et les hommes qui tentent de la faire triompher ébauchent même l'organisation d'un parti autour du général La Fayette ${ }^{28}$.

Après quoi, en février 1801, l'opposition s'organise autour du tribun Bailleul, qui avait pourtant manifesté un réel anti-jacobinisme au moment de Brumaire ${ }^{29}$; il réunit chez lui, autour d'un dîner qui sert de prétexte, des Thermidoriens et d'anciens Conventionnels : "On y a dit qu'un changement dans le gouvernement pourrait seul affranchir la république de l'esclavage $\aleph^{30}$. Bailleul apparaît encore au cœur des groupes d'opposants en janvier 1802. C'est chez lui que se réunissent par exemple Daunou, Benjamin Constant, Chénier ou encore le député des Deux-Nèthes Beerembroeck, bientôt exclu du Corps législatif en raison précisément de la virulence de son opposition : la police relève en effet que dans ces réunions privées, il affirme que le Corps législatif doit être au-dessus de celui qui a le pouvoir de faire exécuter les lois, que tout arrêté du gouvernement tendant à interrompre la marche du Corps législatif est un attentat aux droits du peuple ${ }^{31}$. Les tenants de l'opposition démocratique sont décidément soumis à une active surveillance qui témoigne de ce que l'idée républicaine, dans la pureté de ses principes, inquiète le gouvernement: ceux qui s'y montrent fidèles sont par définition des opposants qui vont bientôt perdre les positions officielles qu'ils étaient parvenus à conserver depuis 1800. Et c'est encore autour de Bailleul qu'en octobre 1802 la police repère les "exclusifs", qui espèrent maintenant qu'une opposition pourra devenir efficace au sein du Sénat ${ }^{32}$.

En dépit du durcissement du régime à partir de 1802, les anciens acteurs de la vie politique ne renoncent pas forcément à exprimer leur opposition. C'est donc une " société de mécontents » qui est identifiée comme se réunissant rue du Bac en février 1802, lorsque s'annonce l'élimination de Beerembroeck. On s'y exprime contre les atteintes aux libertés et contre la renaissance d'une cour, tout en se disant qu'il est impossible que cela dure longtemps : en 1802, les républicains persistent à croire que l'idée républicaine est encore suffisamment vive pour résister à l'évolution du régime. Les archives de la police révèlent d'ailleurs que les acteurs des temps de la Convention montagnarde peuvent encore jouer un rôle important : il en est ainsi de Bonjour, qui a 
été chef de la police secrète du Comité de Salut Public ${ }^{33}$, ou encore de Palis, ancien président du Comité révolutionnaire de la section du Bonnet Rouge ${ }^{34}$. Elles indiquent même l'existence de lignages de républicains : ainsi le fils de l'ex-conventionnel Maure, établi comme fabricant de bas à Paris, est repéré, en décembre 1802, pour organiser des réunions dans lesquelles on prononce des sarcasmes contre le gouvernement ${ }^{35}$. Les sources policières témoignent aussi des liens entre les milieux républicains lyonnais et ceux de Paris: en septembre 1803, on relève la présence de Badin, qui avait été président d'un comité révolutionnaire lyonnais ${ }^{36}$; en octobre, des réunions se tiennent chez Parent, qui fut lui aussi membre d'un comité lyonnais ${ }^{37}$.

11 En fait, l'opposition tentée par les anciens acteurs de la Révolution ne disparait pas totalement avec l'Empire. En avril 1806, deux mémoires contre les impôts indirects ont été répandus dans le département du Pas-de-Calais. Des passages sont considérés comme des provocations à la révolte. Or les auteurs présumés ont joué un rôle entre 1791 et 1799 : l'un, François, était membre de la Législative, l'autre, Debret, a été un haut fonctionnaire de la République, à la tête de l'administration du département du Pas-de-Calais. Est-ce précisément en raison de cette identité que la police prévoit que, dans le cas d'une mise en accusation devant les tribunaux, ces deux hommes seront acquittés? Quoi qu'il en soit, il est décidé d'informer le Grand Juge et de prévoir de simples "mesures de haute police", ce qui signifie tout simplement un emprisonnement arbitraire ${ }^{38}$. On est loin maintenant du temps où la police pouvait se contenter d'écouter les propos hostiles et de les transcrire, en vue de préparer d'éventuels vastes coups de filet. Elle n'est plus dans l'attente mais dans la répression immédiate, preuve finalement de ce que cette opposition est perçue comme un réel danger.

12 Après 1806 , la force des oppositions semble encore diminuer. Les autorités repèrent toutefois une résurgence de ce qu'elles appellent des «mauvais propos » en novembre 1807. On arrête plusieurs individus identifiés comme " anarchistes »" $:$ or, L'Écrivain et Didier ont été membres du tribunal révolutionnaire ${ }^{40}$, Hivert et Gille ont pour leur part appartenu au comité révolutionnaire ${ }^{41}$. Au total, neuf prévenus sont interrogés, il apparaît qu'ils tentaient pour certains d'entre eux de fédérer des "révolutionnaires exagérés ", organisant des réunions à Versailles ${ }^{42}$. Quelques mois plus tard, ils sont finalement placés en résidence surveillée loin de Paris ${ }^{43}$. Après quoi, en juin 1808 , la police a vent du premier complot dans lequel a trempé Malet ${ }^{44}$, arrêté donc une première fois ${ }^{45}$. Son rôle confirme d'ailleurs combien l'armée a constitué un terreau favorable à l'opposition républicaine. Mais les positions résolument républicaines de Moreau ou l'action de Bernadotte, et d'autres officiers, dans le complot jacobin des libelles, dit « des pots de beurre », sont trop connues pour qu'il soit utile d'y revenir ici. Des hommes rompus à l'action politique

13 Le mode d'action de ces hommes est avant tout celui de l'expression sur la place publique. Évoquons pour mémoire leurs menées dans les théâtres ${ }^{46}$. Un rapport, de mars 1801, note que «c'est principalement dans les foyers des théâtres que les malveillants sèment leurs poisons", surtout au Vaudeville ${ }^{47}$. Mais la parole protestataire ne reste pas alors confinée en ces lieux, les opposants s'appliquent bien au contraire à faire circuler nombre de rumeurs. Ils tentent de mobiliser les populations en agitant le spectre d'un retour à l'Ancien Régime, comme en novembre 1800 :

«Ces mêmes exclusifs cherchent par leurs subalternes à répandre l'alarme et le trouble; ils ont fait circuler parmi le peuple qu'une des conditions de la paix serait la déportation de plus de cinq mille républicains connus ; qu'un Bourbon va monter 
sur le trône et mille autres contes qui jettent dans la masse des citoyens une sorte d'inquiétude ${ }^{48}$. misère était extrême dans les départements, qu'elle le deviendrait bientôt aussi à Paris et qu'il fallait s'attendre cet hiver à de grands malheurs. À tous ces propos, ils joignent ordinairement les plus grossières plaisanteries contre l'ordre actuel des choses $»^{51}$. La politique des républicains semble donc être désormais de semer l'inquiétude et d'affirmer à Paris que rien ne va en province afin, espèrent-ils sans doute, de multiplier les protestations et d'encourager les oppositions. Telle apparaît encore leur ligne de conduite en janvier 1803, quand ils répètent «que la république française est perdue, attendu que l'état des finances ne fait qu'empirer $"^{52}$. Ils comptent ensuite sur la guerre pour semer le désarroi et déstabiliser le régime ${ }^{53}$, et la fausse nouvelle devient une arme : ainsi, en août 1803, ils annoncent que l'armée est touchée par une grande vague de désertions ${ }^{54}$, puis ils sèment de nouveau l'alarme au sujet de l'état des finances publiques, ils annoncent également que les départements de l'Ouest seraient dans la plus grande fermentation et que de nombreuses villes auraient été désarmées ${ }^{55}$. Ils tentent également de susciter la révolte en annonçant que le gouvernement va établir des impôts indirects sur le beurre, les œufs et autres denrées de première nécessité56.

Leur but semble être ainsi de soulever les ouvriers des faubourgs, même s'ils se méfient de l'accueil que peut leur réserver le faubourg Saint-Antoine: la police note qu'ils s'appliquent à éviter de trop s'y montrer ${ }^{57}$. Ils semblent préférer agir par exemple lorsque la foule se rassemble lors de défilés militaires, ils affectent alors, comme en septembre 1803, quand il est question de l'invasion de l'Angleterre, de plaindre les troupes et ils disent que le gouvernement va les sacrifier ${ }^{58}$. Mais ils persistent alors à recourir aux bruits d'insurrection provinciale, annonçant cette fois que c'est le Midi qui serait en pleine révolte, prédisant que, donc, la cause des patriotes ne peut plus 
manquer de triompher ${ }^{59}$, mais mettant aussi en garde pour le cas où l'on tarderait à agir : ils mêlent en effet au bruit précédent celui de la préparation d'un massacre ou d'un emprisonnement de tous les patriotes encore libres ${ }^{60}$. Dans les modalités de diffusion de nouvelles alarmistes destinées à mobiliser leurs partisans, les républicains ont donc recours aux réflexes de peur que peuvent mobiliser les souvenirs de certaines heures de la Révolution: ils se placent ainsi décidément dans une dynamique qui se trouve à la frontière du champ du secret et de l'espace du public. Et c'est encore cela qui est à l'œuvre quand, en août 1804, les «malveillants" répandent des bruits alarmistes sur la situation des finances et de l'armée et sur la position diplomatique de la France ${ }^{61}$. Et c'est également cela qu'ils recherchent quand la police note que les « anarchistes » tentent d'obtenir des places pour assister au couronnement ${ }^{62}$.

Ils ne négligent toutefois pas la diffusion de leurs idées par l'écrit. Des libelles sont préparés dans une imprimerie clandestine des environs de Paris ${ }^{63}$, dont un pamphlet intitulé "Pétition au Tribunat " ${ }^{64}$. L'auteur est finalement arrêté en décembre 1803 : il s'agit d'un ancien géomètre arpenteur devenu libraire, nommé Aubry ${ }^{65}$. En même temps que lui, la police intercepte l'officier de santé Guérin, qui avait contribué à la diffusion de ce libelle. Ces deux arrestations ont d'ailleurs profondément découragé les républicains encore en liberté ${ }^{6}$, affaiblis quelques jours plus tard par l'arrestation de Bonjour ${ }^{67}$. Et même si, à la fin de décembre 1803, ils persistent à dire que leur mouvement est encore vigoureux et que les meilleures têtes n'ont pas été prises ${ }^{68}$, l'année 1804 les voit découragés : en avril 1804, alors que se prépare la proclamation de l'Empire, ils reconnaissent, selon la police «que l'opinion générale, surtout celle du peuple, est en faveur du premier consul et qu'on ne peut révoquer en doute l'attachement qu'on lui porte ». Ils ajoutent «que cet état de choses n'est pas propre à leur donner des espérances et que l'apathie et l'insouciance des habitants des faubourgs finit par les décourager tout à fait $\aleph^{69}$. Découragement accentué lorsqu'ils apprennent que la proclamation de l'Empire est officiellement acceptée par les généraux Masséna et Bernadotte ${ }^{70}$. En septembre 1804, ils ne se réunissent même pas pour célébrer la proclamation de la République ${ }^{71}$. La police repère bien encore quelques réunions à l'automne de $1804^{72}$, mais tous se refusent à être témoins des cérémonies du couronnement : « C'est fini pour nous, disent-ils alors - selon la police - il n'y a plus de liberté $»^{73}$.

Pourtant, il existe encore quelques activistes pour croire à l'efficacité, au début de l'Empire, d'actions ambitieuses. Le découragement des républicains n'est pas aussi absolu que les autorités semblent le croire. Ainsi, en mars 1805, le sous-préfet de Poligny, dans le Jura, est informé de ce qu'une réunion d'«exclusifs»- le terme réapparait alors - aurait pour but apparent le rétablissement des Bourbons, mais pour objectif réel « le règne de 1793 ». Huit mille hommes seraient prêts à se mobiliser, qui prévoient notamment de s'en prendre à l'empereur s'il passe par le Jura en revenant d'Italie ${ }^{74}$. En fait, un véritable soulèvement devait, à en croire les rumeurs, embraser à la fois la Bretagne et la Franche-Comté ${ }^{75}$. Informations prises, les meneurs républicains s'avèrent n'être que six, "n'ayant aucun moyen pécuniaire, ni considération personnelle $\aleph^{76}$, deux d'entre eux, Declancher et Pyraud, ont surtout été repérés pour avoir tenu des propos hostiles au gouvernement dans un café de Besançon. Voilà qui donne le sentiment que cette opposition devient clairsemée.

Une survivance dans l'isolement? 
20 Les rapports de police se font donc bientôt beaucoup moins diserts sur le sort de tels opposants, la rubrique "exclusifs " n'apparaît plus, tout semble indiquer que les dangers d'une opposition républicaine s'estompent. Il est vrai que les figures célèbres autour desquelles se rassemblaient encore les républicains rentrent peu à peu dans le rang: il en est ainsi de Masséna, ou de Bernadotte qui a pourtant été, semble-t-il, l'instigateur du « complot des libelles » ou " des pots de beurre » en 1802 et avec lui des seconds couteaux comme Edouard-François Simon ou bien encore le frère de Marbot ${ }^{77}$. Moreau est pour sa part exilé aux États-Unis, Benjamin Constant et Germaine de Staël sont en Suisse, Sieyès s'efface ${ }^{78}$, de même, en fait, que Carnot, Bailleul ou Marie-Joseph Chénier; La Fayette se retire à la campagne, Daunou s'enferme dans ses fonctions d'archiviste, Jean-Baptiste Say dans celles d'un industriel du Pas-de-Calais. Est-ce à dire que le mouvement républicain est dès lors défunt?

21 Reconnaître cela serait oublier comment Garat, Boissy d'Anglas, Destutt de Tracy, Volney, Lanjuinais, Jacquemont ou encore Cabanis conservent leurs sympathies à l'idée républicaine, ce serait aussi oublier que les tentatives de Malet en 1808 puis en 1812 témoignent également de la persistance des espoirs des démocrates ${ }^{79}$. Nous ne reviendrons pas ici sur ce que celles-ci apprennent des fidélités républicaines puisque cela a déjà été fait par Bernard Gainot ${ }^{80}$, y compris sur les rôles de Jacques-Rigomer Bazin, de Pierre-Alexandre Lemare ou encore de Buonarroti. Du reste, ces conspirations semblent attester la force des actions de la Société des Philadelphes, matrice des sociétés secrètes et protectrice de ces réseaux républicains dans lesquels agit l'aristocrate révolutionnaire Antonelle ${ }^{81}$. Considérer le mouvement républicain comme défunt, ce serait enfin faire fi de la façon dont l'idée républicaine a pu subsister en deçà des cercles des activistes politiques, ne serait-ce que dans la mémoire des hauts faits de la Révolution. Ainsi, en septembre 1804, c'est le groupe des vainqueurs de la Bastille qui fait entendre sa voix, et de façon fort symbolique puisqu'il se permet d'entonner publiquement la Marseillaise ${ }^{82}$. Reste que, parcourant quelques jours plus tard, toujours fort bruyamment, les rues du faubourg Saint-Antoine, ils auraient été fort mal accueillis dans les cabarets « à cause de leur jactance et de leurs propos ${ }^{83}$.

Par ailleurs, on repère dès les débuts du Consulat que l'idée républicaine est alors aussi portée par des hommes isolés. L'expression d'une hostilité au nouvel ordre de choses contrastant avec l'ancien ordre républicain a aussi été le fait de personnes agissant seules. Ainsi, en mai 1801, un homme assiste au départ du premier consul pour le spectacle et exprime sa désapprobation face à l'apparat dont il le voit entouré : "Des gardes à pied, des gardes à cheval, un peuple immense pour le voir monter en voiture ». Après quoi il partit en fredonnant : "ça ne durera pas toujours car cela finira bientôt ». La filature échoue, mais la police ne renonce pas pour autant à le chercher ${ }^{84}$. Le ton même sur lequel ces mots sont prononcés confirme une fois de plus que l'opposition s'exprime à la frontière du privé et du public : l'homme ne cherche que timidement à se faire entendre alentour. Mais d'autres s'expriment plus résolument: en Vaucluse, en 1801 également, Noël Bertrand, cultivateur, est arrêté pour avoir chanté une chanson faisant l'éloge de la Révolution et de Robespierre. Il s'est même battu avec le brigadier qu'il a croisé et qui pour sa part a traité Robespierre de "polisson " ${ }^{85}$. Il semble bien que ce soit aussi de façon assez isolée, loin en tout cas de tout réseau d'envergure, qu'a agi un jeune garçon tailleur de 24 ans nommé Léon : il avait placardé aux Tuileries des affiches « contenant des mots injurieux sur les circonstances actuelles ». Ces affiches ont été apposées le 5 mai 1804, alors que le célèbre discours de Carnot contre 
l'établissement de l'Empire a été prononcé au Tribunat quelques jours plus tôt, le 30 avril. Le jeune homme a reconnu avoir lui-même déposé d'autres exemplaires de cette affiche dans différents quartiers de Paris, y compris à proximité de l'hôtel particulier de Joseph Bonaparte. Son seul complice semble être celui qui a écrit les placards : il n'est âgé, lui, que de 19 ans, il est également tailleur et se nomme Poinet. Qualifiés tous deux d'« exclusifs renforcés", ils sont par ailleurs reconnus par la police comme de la " canaille » qui «n'est dirigée par personne ${ }^{86}$, ce qui permet de dédramatiser et de dépolitiser de tels épisodes pourtant récurrents. Ce jeune républicain ne laisse pas d'autre trace, pour l'heure, à l'historien: sans doute a-t-il été, comme d'autres, emprisonné plusieurs mois, voire plusieurs années sans autre forme de procès.

En janvier 1805, Philippe Ignard est arrêté pour avoir fait imprimer clandestinement, en mille exemplaires, une adresse aux Français les invitant à renverser le gouvernement. Ce marchand de sel a été maire de sa commune de Tresson (Sarthe) en 1793 ; il est identifié par la police comme « un fanatique démagogue qui a conservé ses opinions de 1793 ». Il affirme avoir agi seul, être le seul auteur de cette adresse qu'il avait l'intention de répandre dans Paris mais aussi dans les départements. Un semblable événement intervient quelques jours plus tard à Toulon : en février 1805, un placard y est trouvé sur les murs de la préfecture maritime. Il visait à rappeler à la troupe les temps où elle remportait des victoires pour la cause de la liberté et invitait le peuple à se soulever le soir même, à l'occasion d'une fête organisée pour le couronnement italien. Les autorités locales mettent ce placard sur le compte des agissements « des partisans du régime de 1793 », tout en précisant qu'il n'aurait pour auteur qu'un homme isolée ${ }^{87}$. Si les sources ne précisent pas ici s'il s'agit de placards manuscrits ou imprimés, il est en revanche avéré que Philippe Ignard a pour sa part recours à une préparation matérielle qu'il faut dissimuler: l'apprentissage de la politique passe donc alors pour certains par la maîtrise de l'action clandestine. Les degrés d'investissement dans l'expression des fidélités républicaines sont donc variables, mais tous révèlent les formes d'une acculturation politique par delà 1799 .

Quoi qu'il en soit, il existe une résistance sans organisation véritable, dont on retrouve trace tout au long de l'Empire. Ainsi Vauquelin, tisserand, et Quillet, chapelier, sont arrêtés dans le Calvados en mars 1810, pour avoir tenté de dresser la population contre les droits réunis ${ }^{88}$. Mais il est vrai qu'il n'est plus guère question de les identifier comme « républicains", ni même comme "exclusifs». En creux, les rapports de police montrent que le régime s'emploie décidément à dépolitiser les oppositions en les identifiant comme des actes isolés, mais aussi en les soustrayant souvent à la justice. Ce qui n'empêche pas les autorités policières de reconnaître parfois, comme à propos de Toulon en mai 1805, qu'il subsiste un parti des émigrés et un camp « des anarchistes regrettant le régime de $1793 »^{89}$, situation identique à celle qui règne alors également à Marseille, où « les révolutionnaires exagérés sont contenus avec soin $»^{90}$, situation qui semble encore réelle à Toulon en $1808^{91}$. Ainsi, l'idée de l'isolement des fidélités républicaines est certes diffusée par la police, mais cela cache peut-être une survivance plus dense qu'on ne l'a longtemps cru.

L'on doit d'ailleurs s'interroger sur le devenir des idées républicaines quand on constate que la plupart des opposants sont arrêtés pour "propos injurieux contre l'empereur ». Ces mentions qui apparaissent dans les rapports de police, avec une constante imprécision, sont révélatrices de ce que les autorités répugnent à relever le détail des mots. Mots indicibles? Intransmissibles par écrit? Ou simplement trop 
confus pour être transcrits, mais assez précis pour susciter une arrestation puis un maintien en détention par mesure de haute police. Car tel fut le sort de la plupart de ceux qui ont été ainsi pris en flagrant délit d'opposition républicaine, tel fut le destin des opposants à une république hypocritement confiée à un consul puis à un empereur, telle fut la voie suivant laquelle le projet républicain a été éliminé du champ politique français durant les années napoléoniennes.

Toutefois, les propos séditieux notés par les policiers, en dépit du caractère impressionniste des rapports, attestent que la référence politique, pour ces humbles citoyens privés de toute voie légale d'expression, est la personne de l'empereur et non la République. Face à cette litanie des oppositions ainsi exprimées, il semble bien que la République ne fait guère sens que pour quelques membres d'une élite intellectuelle prête à agir jusque dans des complots. En fait, les Français demeurent nombreux à accepter l'idée d'un mode de gestion de la Cité dans lequel tout dépend des actes de la personne du souverain. Ainsi, le 16 janvier 1813, un placard trouvé à Fontainebleau indique " Nous demandons à notre bon empereur la diminution du pain à 3 sols la livre,

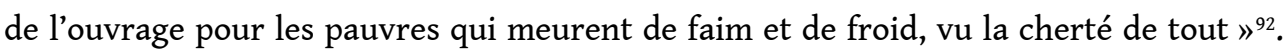
Les oppositions s'expriment d'ailleurs aussi par des appels à cesser de servir l'empereur, tels que le précisent par exemple deux écrits anonymes trouvés en décembre 1812 sous la porte du maire du village de Clermont, près de Verdun ${ }^{93}$. L'urgent, pour les opposants, n'est pas d'appeler à une résurrection républicaine mais de convaincre de la nécessité de renverser le régime impérial, tout en appelant, en l'occurrence, à la diminution du prix du pain.

Mais l'idée républicaine ne demeure-t-elle pas implicite dans l'opposition de tous ceux qui se dressent contre la conscription, allant jusqu'à la rébellion contre des gendarmes pour délivrer des conscrits emmenés de force? Et puis n'est-ce pas rester fidèle à l'esprit républicain que d'orchestrer des cérémonies d'injures contre la Légion d'honneur ${ }^{94}$. N'en est-il pas de même de ceux qui tentent de dresser la population contre les employés des droits réunis? En septembre 1800, à l'occasion de l'établissement de nouveaux droits d'octroi, ceux que la police qualifie entre autres d'« exclusifs " «crient au retour de l'Ancien Régime » et insinuent que le régime va ainsi peu à peu rétablir " tous les anciens usages " $^{95}$. Des fidélités républicaines existent donc, incontestablement, dans l'anonymat des formes de politisation des humbles, tout autant que dans les termes du débat politique des joutes oratoires parisiennes et parlementaires de plus en plus rares, tout autant, également, que dans les réseaux élaborant de retentissants complots.

\section{NOTES}

1.Mentionnons toutefois le travail de Jean VIDALENC, « L'opposition sous le Consulat et l'Empire ", Annales historiques de la Révolution française, 1968, n 194, p. 472-488. 
2.Figurée par exemple en 1849 sur le célèbre Enterrement à Ornans de Courbet : voir Jean-Luc MAYAUD, Courbet, L'Enterrement à Ornans. Un tombeau pour la République, Paris, La Boutique de l'Histoire, 1999.

3.Michel Vovelle, Les Jacobins. De Robespierre à Chevènement, Paris, La Découverte, 2001 (1ère édition en italien en 1998), p. 107.

4.Eugenio Di RIENzo, L'Aquila e il berretto frigio. Per una storia del movimento democratico in Francia da Brumaio ai Cento giorni, Napoli, Edizioni Scientifiche Italiane, 2001 ; Georges FOURNIER, « La longue survie du jacobinisme toulousain du Directoire à la Restauration », dans Christine LE BOZEC, Éric WAUTERs (dir.), Pour la Révolution française, Rouen, Publications de l'Université de Rouen, 1998, p. 365-370 ; Bernard GAINOT, « Un itinéraire démocratique post-thermidorien : Bernard Metge », dans Christine LE BOZEC, Éric WAUTERS (dir.), Pour la Révolution française, op. cit., p. 93-106 ; Bernard GAINOT, 1799, un nouveau jacobinisme?, Paris, CTHS, 2001, 542 p. ; Bernard GAINOT, « Pratiques politiques et stratégies narratives. Hypothèses de recherche sur les conspirations militaires : "La conspiration Malet" de 1808 », dans Politix, n 54, p. 93-117 ; Bernard GAINOT, «Réflexion sur une forme politique de transition. À propos de la conspiration Malet de 1808 ", dans La plume et le sabre. Mélanges offerts à Jean-Paul Bertaud, Paris, Publications de la Sorbonne, 2002, p. 513-524 ; Pierre SERNA, Antonelle, aristocrate révolutionnaire, 1737-1817, Paris, Éditions du Félin, 1997 ; Pierre SERNA, « Rigomer Bazin et la Restauration : penser la république dans la monarchie ", Annales historiques de la Révolution française, $\mathrm{n}^{\circ} 325,2001$, p. 53-76.

5.Michel Vovelle, Les Jacobins, op. cit., p. 108.

6.Voir entre autres : AN, F7 3829-3832. Les termes d' « exagérés », d'« exaltés », d'« anarchistes » sont ceux que Fouché emploie dans ses Mémoires pour désigner les Jacobins : Michel Vovelle, Les Jacobins, op. cit., p. 107.

7.Je remercie très vivement Marc Deleplace des discussions que nous avons eues sur ce sujet. Sur l'utilisation du mot anarchiste pour désigner les jacobins, voir notamment Marc DelePlace, L'Anarchie, de Mably à Proudhon (1750-1850). Histoire d'une appropriation polémique, Paris, ENS éditions, 2000.

8.Pierre SERNA, La République des girouettes (1789-1815... et au-delà). Une anomalie politique[20: la France de l'extrême centre, Paris, Champ Vallon, 2005.

9.AN, F7 3829, rapport de la préfecture de police du 4 vendémiaire an IX (26 septembre 1800).

10.Jean Destrem, « Documents sur les déportations du Consulat », Revue historique, maijuin 1878, p. 78-120.

11.AN, F7 3829, rapport du 13 brumaire an IX (4 novembre 1800).

12.Louis De Villefosse, Janine Bouissounouse, L'opposition à Napoléon, Paris, Flammarion, 1969, p. 158. Mais pour le reste, notons que cet ouvrage ne permet guère d'en savoir davantage sur le sort des républicains sous l'Empire.

13.AN, F7 3829.

14.Michael D. SiBALIS, « Un aspect de la légende noire de Napoléon : le mythe de l'enfermement des opposants comme fous », Revue de l'Institut Napoléon, 1991-I, n 156, p. 9-24; « Prisoners by Mesure de Haute Police under Napoleon I : Reviving the Lettres de cachet ", in Proceedings of the Annual Meeting of the Western Society for French History, volume 18, 1991, p. 261-269; « Arbitrary detention, human rights and the Napoleonic Senate ", dans Howard G. Brown, Judith A. Miller, Taking liberties, Problems of a New Order 
from the French Revolution to Napoleon, Manchester and New York, Manchester University Press, 2002, p. 166-184.

15.AN, F7 3830, rapport du 30 pluviôse an X (19 février 1802).

16.Voir par exemple : AN, F7 3830, à partir de thermidor.

17.AN, F7 3830, rapports des 15 et 17 fructidor an X (2 et 4 septembre 1802).

18.AN, F7 3831, rapport du 6 ventôse an XI (25 février 1803).

19.AN, F7 3831, rapport du 8 messidor an XI (27 juin 1803).

20.AN, F7 3831, rapport du $5^{\mathrm{e}}$ jour complémentaire de l'an XI (22 septembre 1803).

21.AN, F7 3832, rapport du 20 vendémiaire an XII (13 octobre 1803).

22.Idem.

23.AN, F7 3832, rapport du 8 brumaire an XII (31 octobre 1803).

24.AN, F7 3832, rapports des 14, 16, 22 brumaire an XII (6, 8 et 14 novembre 1803).

25.Je renvoie une fois pour toutes, pour l'Empire, aux versions publiées des bulletins de police : Ernest d'HAUTERIVE, La police secrète du Premier Empire. Bulletins quotidiens adressés par Fouché à l'empereur, Paris, Perrin puis Clavreuil, 1908-1964, 5 volumes, puis Nicole GOTTERI, La police secrète du Premier Empire. Bulletins quotidiens adressés par Savary à l'empereur, Paris, Champion, 1997-2004, 7 volumes.

26.Notice « Jacobins » par Jean TULARD, Dictionnaire Napoléon, Paris, Fayard, 1986.

27.Précisons toutefois que la publication des bulletins de Savary souffre de l'absence du précieux index analytique qui complétait la publication des bulletins de Fouché.

28.AN, F7 3829, rapport du 7 frimaire an IX (28 novembre 1800).

29.Notice «Bailleul » par Jean TULARD dans le Dictionnaire Napoléon, op. cit.

30.AN, F7 3829, rapport du 5 ventôse an IX (24 février 1801).

31.AN, F7 3830, rapport du 21 nivôse an X (11 janvier 1802).

32.AN, F7 3831, rapport du 10 vendémiaire en XI (2 octobre 1802).

33.AN, F7 3832, 25 frimaire an XII (17 décembre 1803).

34.AN, F7 3832, 28 frimaire an XII (20 décembre 1803).

35.AN, F7 3831, rapport du 29 frimaire an XI (20 décembre 1802).

36.AN, F7 3832, rapport du 1er vendémiaire an XII (24 septembre 1803).

37.AN, F7 3832, rapport du 25 vendémiaire an XII (18 octobre 1803).

38.AN, F7 3090, rapport du 4 avril 1806.

39.Bulletin de police du 6 novembre 1807 .

40.Bulletin de police du 7 novembre 1807.

41. Bulletin de police du 10 novembre 1807 .

42.Bulletin de police du 14 novembre 1807 .

43.Bulletin de police du 11 avril 1808 .

44.Bulletin de police du 9 juin 1808.

45.Bulletin de police du 10 juin 1808 .

46.Cyril TRIOLAIRE, « Contrôle social et arts du spectacle en province pendant le

Consulat et l'Empire. L'exemple du Puy-de-Dôme », Annales historiques de la Révolution

française, $n^{\circ} 333,2003$, p. 45-66.

47.AN, F7 3829, rapport du 23 ventôse an IX (14 mars 1801).

48.AN, F7 3829, rapport du 13 brumaire an IX (4 novembre 1800).

49.AN, F7 3830, rapport du 25 frimaire an X (16 décembre 1801).

50.AN, F7 3831, rapport du 6 brumaire an XI (28 octobre 1802).

51.AN, F7 3831, rapport du 28 vendémiaire an XI (20 octobre 1802).

52.AN, F7 3831, rapport du 12 nivôse an XI (2 janvier 1803).

53.AN, F7 3831, rapport du 5 germinal an XI (26 mars 1803). 
54.AN, F7 3831, rapport du 1er fructidor an XI (19 août 1803).

55.AN, F7 3831, rapport du 8 fructidor an XI (26 août 1803).

56.AN, F7 3831, rapport du 15 fructidor an XI (2 septembre 1803).

57.AN, F7 3831, rapport du 5e jour complémentaire de l'an XI (22 septembre 1803).

58.AN, F7 3832, rapport du 1er vendémiaire an XII (24 septembre 1803).

59.AN, F7 3832, rapport du 25 vendémiaire an XII 18 octobre 1803).

60.AN, F7 3832, rapport du 27 vendémiaire an XII (20 octobre 1803).

61.Bulletin de police du 30 août 1804 .

62.Bulletin de police du 15 octobre 1804 .

63.AN, F7 3832, rapports de vendémiaire et de brumaire an XII.

64.AN, F7 3832, rapport du 22 brumaire an XII (14 novembre 1803).

65.AN, F7 3832, rapport du 25 frimaire an XII (17 décembre 1803).

66.AN, F7 3832, 26 frimaire an XII (18 décembre 1803).

67.Idem.

68.AN, F7 3832, 9 nivôse an XII (31 décembre 1803).

69.AN, F7 3832, 9 floréal an XII (29 avril 1804).

70.AN, F7 3832, 23 floréal an XII (13 mai 1804).

71.AN, F7 3833, 2 vendémiaire an XIII (24 septembre 1804).

72.AN, F7 3833, 12, 22 vendémiaire an XIII (4 et 14 octobre 1804).

73.AN, F7 3833, 14 frimaire an XIII (5 décembre 1804).

74.Bulletin de police du 27 mars 1805.

75.Bulletin de police du 3 avril 1805.

76.Bulletin de police du 12 avril 1805.

77.Voir les notices au nom de ces hommes dans le Dictionnaire Napoléon, op. cit.

78.Jean-Denis BREDIN, Sieyès, la clef de la Révolution française, Paris, Éditions de Fallois, 1988, $607 \mathrm{p}$.

79.Louis DE VILLEFOSSE, Janine BOUISSOUNOUSE, op. cit., p. 308.

80.Articles cités note 3 , supra.

81.Pierre SERNA, Antonelle, op. cit.

82.AN, F7 3832, 17 fructidor an XII (4 septembre 1804).

83.AN, F7 3832, 28 fructidor an XII (15 septembre 1804).

84.AN, F7 3829, rapport du 3 prairial an IX (23 mai 1801).

85.AD Vaucluse, 3U2/926. Registre de jugement en correctionnel du tribunal de première instance d'Avignon, cité dans Hélène MARCHESI, La contestation de l'ordre établi : les oppositions à l'autorité de l'État sous le Consulat et l'Empire dans les arrondissements d'Avignon et d'Orange, mémoire de maîtrise sous notre direction, Université d'Avignon, 2004, p. 84.

86.AN, F7 3832, 15 floréal an XII (5 mai 1804).

87. Bulletin de police du 1er février 1805 .

88.AN, F7 3091, rapport du 23 mars 1810.

89.Bulletin de police du 3 mai 1805.

90.Bulletin de police du 20 août 1805 .

91.Bulletin de police du 23 juin 1808.

92.AN, AF IV 1525, rapport du 3 février 1813.

93.Bulletin de police des 1-2 janvier 1813 .

94.Bulletin de police du 11 mars 1805.

95.F7 3829, rapport de la préfecture de police du 4 vendémiaire an IX (26 septembre 1800). 


\section{RÉSUMÉS}

Les historiens du Premier Empire ont pour beaucoup négligé de s'interroger sur les survivances républicaines après 1799. Même les évocations nombreuses du coup d'État de Malet, dans une historiographie classique, ne font guère référence à l'influence, dans cet événement, des fidélités républicaines. Pourtant, les recherches récentes comme les sources policières permettent de mettre en évidence le fait que ces oppositions ne disparaissent pas totalement de la vie politique sous l'Empire. L'étude de ces survivances permet d'ailleurs de prendre conscience non seulement du rôle d'acteurs des temps révolutionnaires condamnés à agir désormais souvent dans le secret, mais aussi de la place des anonymes dans les oppositions relevant des nostalgies républicaines. Une telle étude montre de plus à quel point les persistances d'une vie politique se trouvent à la frontière entre espace public et espace secret.

Republican Loyalties under the Consulate and Empire. Historians of the First Empire have much neglected the study of the persistance of republicanism after 1799. Even the numerous references to the coup d'Etat of Malet, in the classic historiography, scarcely allude to the influence in this event of republican loyalties. Recent research using police sources, however, reveals that political opposition did not completely disappear from political life under the Empire. The study of this continuity of allegiances not only demonstrates the role of political personalities in revolutionary times, often condemned to operate later in secret, but also of the role of anonymous persons forming diverse oppositions imbued with republican nostalgia. Such a study, moreover, shows to what extent the persistance of a political life is found at the border between public and secret space.

INDEX

Mots-clés : jacobins, Premier Empire, oppositions politiques, police, espace public, secret

\section{AUTEUR}

\section{NATALIE PETITEAU}

Université d'Avignon, UFR Lettres et sciences humaines, 74 rue Louis Pasteur, 84029 Avignon Cedex, natalie.petiteau@wanadoo.fr 